\title{
Engraftment of Cells from Porcine Islets of Langerhans and Normalization of Glucose Tolerance Following Transplantation of Pig Pancreatic Primordia in Nonimmune-Suppressed Diabetic Rats
}

\author{
Sharon A. Rogers, ${ }^{*}$ Thalachallour Mohanakumar, ${ }^{\dagger \neq}$ \\ Helen Liapis, ${ }^{\ddagger}$ and Marc R. Hammerman ${ }^{\star \S}$ \\ From the Renal Division, Departments of Medicine," Surgery, ${ }^{\dagger}$ \\ Patbology and Immunology, ${ }^{\ddagger}$ and Cell Biology and Physiology, \\ Washington University School of Medicine, St. Louis, Missouri
}

Transplantation therapy for human diabetes is limited by the toxicity of immunosuppressive drugs. However, even if toxicity can be minimalized, there will still be a shortage of human donor organs. Xenotransplantation of porcine islets may be a strategy to overcome these supply problems. Xenotransplantation in mesentery of pig pancreatic primordia obtained very early during organogenesis [embryonic day 28 (E28)] can obviate the need for immune suppression in rats or rhesus macaques. Here, in rats transplanted previously with E28 pig pancreatic primordia in the mesentery, we show normalization of glucose tolerance in nonimmune-suppressed streptozotocin-diabetic LEW rats and insulin and porcine proinsulin mRNA-expressing cell engraftment in the kidney following implantation of porcine islets beneath the renal capsule. Donor cell engraftment was confirmed using fluorescent in situ hybridization for the porcine $X$ chromosome and electron microscopy. In contrast, cells from islets did not engraft in the kidney without prior transplantation of E28 pig pancreatic primordia in the mesentery. This is the first report of prolonged engraftment and sustained normalization of glucose tolerance following transplantation of porcine islets in nonimmune-suppressed, immunecompetent rodents. The data are consistent with tolerance induction to a cell component of porcine islets induced by previous transplantation of E28 pig pancreatic primordia. (Am J Pathol 2010, 177:854-864; DOI: 10.2353/ajpath.2010.091193)

In that pigs are plentiful and because porcine insulin works well in humans, the pig has been suggested to be a pancreas organ donor for humans with diabetes. The severity of humoral rejection due to pre-existing natural antibodies effectively precludes their use as whole pancreas donors in nonhuman primates or humans. ${ }^{1-4}$ However, isolated islets of Langerhans (islets) can be transplanted into nonhuman primates ${ }^{2,3}$ or humans with diabetes ${ }^{1}$ without initiating humoral rejection. Unfortunately, recent experience with pig to primate islet ${ }^{2}$ or neonatal islet ${ }^{3}$ transplantation shows that sustained insulin independence can be achieved, but only through the use of immune suppressive agents that are not approved for human use or would result in an unacceptable level of morbidity in humans. ${ }^{2,3}$

We have shown that glucose tolerance can be normalized in streptozotocin (STZ)-diabetic (type 1) LEW rats or ZDF (type 2) diabetic rats within 4 weeks following transplantation in mesentery of pig pancreatic primordia obtained very early during embryogenesis [on embryonic day 28 (E28) - just after the organ differentiates and before the time dorsal and ventral anlagen fuse] without host immune suppression. ${ }^{5-8}$ No rat insulin can be detected in STZ-treated rats. Porcine insulin circulates posttransplantation of E28 pig pancreatic primordia (embryonic pancreas) and levels increase after a glucose load. ${ }^{6,7}$ Cells expressing insulin and porcine proinsulin mRNA with $\beta$ cell morphology engraft in host mesentery, mesenteric lymph nodes, liver, and pancreas posttransplantation. ${ }^{5-8}$ Cells originating from E28 pig pancreatic primordia engraft similarly in nonimmune-suppressed STZ-diabetic rhesus macaques. ${ }^{9}$

Long-term engraftment of cells originating from E28 pig pancreatic primordia in non-immune suppressed immune competent hosts reflects tolerance to a cellular

Supported by George M. O'Brien Center DK079333 by grant 1-2008-37 from Juvenile Diabetes Research Foundation (M.R.H.).

Accepted for publication April 2, 2010.

Address reprint requests to Marc R. Hammerman, M.D., Renal Division, Box 8126, Department of Medicine, Washington University School of Medicine, 660 S. Euclid Ave., St. Louis, MO 63110. E-mail: mhammerm@ dom.wustl.edu. 
component present in the primordia or that differentiates in situ following implantation. ${ }^{5-9}$ To ascertain whether tolerance might extend to the same or similar cell component present in porcine islets from adult swine (adult islets), we implanted adult porcine islets beneath the renal capsule of rats that previously had been transplanted with E28 pig pancreatic primordia in mesentery but remained glucose intolerant. Intact porcine islets do not engraft following renal subcapsular implantation. However, a population of cells originating from donor islets with $\beta$ cell morphology that express insulin and porcine proinsulin mRNA engraft in kidneys of rats transplanted previously with E28 pig pancreatic primordia. Glucose tolerance is normalized in these animals. Our observations are consistent with induction of tolerance to a cell component of adult porcine islets by previous transplantation of E28 pig pancreatic primordia in rats.

\section{Materials and Methods}

\section{Induction of Diabetes Mellitus and Treatment with Exogenous Insulin}

Diabetes mellitus was induced in female LEW rats by a single i.p. injection of $40-50 \mathrm{mg} / \mathrm{kg} \mathrm{STZ}$. Rats were considered diabetic if fasting blood glucose levels were 300 $\mathrm{mg} / \mathrm{dl}$ or above 5 consecutive days after STZ administration, at which time E28 pig pancreatic primordia were implanted. Maintenance of glucose levels $<250 \mathrm{mg} / \mathrm{dl}$ during the first 2-3 weeks posttransplantation is necessary to permit optimal differentiation, proliferation, and function of transplanted E28 pig pancreatic primordia. ${ }^{8}$ Accordingly, rats transplanted with pig pancreatic primordia or islets were treated with 1-2 units sq b.i.d. of Lantus insulin (Sanofi-Aventis, Bridgewater, NJ) for 2-3 weeks after implantation to maintain fasting glucose levels, measured every 3 days, at $200-250 \mathrm{mg} / \mathrm{dl}^{8}{ }^{8}$

\section{Isolation and Transplantation of Pancreatic Primordia}

At 28 days gestation, pregnant Yorkshire pigs (Oak Hill Genetics, Ewing, IL) were intubated and anesthesia maintained by inhalation of isoflurane and $\mathrm{O}_{2}$ to effect. The uterus was removed, and the donor pig was then euthanized. Pancreatic primordia from E28 pig embryos were surgically isolated under a dissecting microscope. After isolation, primordia were placed immediately into ice-cold Dulbecco's modified Eagle's Medium:Ham's F12 containing iron-saturated transferrin $(5 \mu \mathrm{g} / \mathrm{ml}), 25 \mathrm{nmol} / \mathrm{L}$ prostaglandin $\mathrm{E}_{1}$ and, $5 \mu \mathrm{g} / \mathrm{ml}$ recombinant human vascular endothelial growth factor (Genentech, South San Francisco, CA) and $10^{-8} \mathrm{M}$ recombinant human Hepatocyte Growth Factor (HGF) (Upstate Biotechnology, Lake Placid, NY). After 45 minutes, six to eight pancreatic primordia were implanted between layers of mesentery of 6- to 10-week-old STZ-diabetic female LEW rat hosts in close proximity to blood vessels. Rats subjected to sham surgery had mesentery prepared for transplantation, but no E28 primordia were transplanted. Host rats received no immune suppression. ${ }^{5-8}$

\section{Isolation and Transplantation of Porcine Islets of Langerhans}

Porcine islets of Langerhans were isolated from female Yorkshire pigs exactly as previously described by one of us (T.M.). ${ }^{10}$ Five thousand islets equivalents (islets), suspended in medium 199 (Sigma-Aldrich, St. Louis, MO) were implanted beneath the renal capsule of STZ-diabetic rats using the technique we described for transplantation beneath the renal capsule of kidney primordia. ${ }^{11}$ Unless otherwise noted, rats had been transplanted in mesentery with E28 pig pancreatic primordia 8 weeks previously. In preliminary experiments using STZ-diabetic LEW rats, the fasting blood glucose levels and glucose tolerance of which had been normalized within 4 weeks of transplantation of E28 pig pancreatic primordia, ${ }^{5,6}$ subsequent implantation of islets in kidney resulted in severe hypoglycemia and death. Accordingly, for islet implantation studies reported here, we used rats, glucose levels in which were reduced by transplantation of E28 pig pancreatic primordia but not to levels in nondiabetic animals. Approximately one in three transplanted rats will manifest such a partial response. ${ }^{9}$ Glucose tolerance can be normalized in such animals by retransplantation of E28 pig pancreatic primordia. ${ }^{9}$ However, levels of fasting blood glucose do not normalize over time if no further transplantation is performed.

\section{Glucose, Insulin, and Rat C Peptide Measurements and Statistics}

Levels of glucose were measured using the Hemocue B-glucose Analyzer (Hemocue, Lake Forest, CA) in whole blood obtained (via tail vein) at $8 \mathrm{AM}$ after an overnight fast. Insulin in serum was measured using one of two enzyme-linked immunosorbent assays (ELISAs): 1) A porcine insulin-specific ELISA (catalog number K6219; DakoCytomation, Carpinteria CA; sensitivity 0.02 ng/ml) that does not detect rat insulin was used to generate data shown in Figure 1, D and F. Insulin measured using this ELISA is designated porcine insulin; or 2) an ultrasensitive ELISA (Mercodia, Winston-Salem, NC; NVC 10-113701 , sensitivity $0.01 \mathrm{ng} / \mathrm{ml}$ ) that detects rat insulin, but cross-reacts with porcine insulin was used to generate data from rats into which no pig tissue is present shown in Figure 1B. Insulin measured using this ELISA is designated rat insulin. Levels of C-peptide were measured using an ELISA specific for rat (number 10-1172-01; Mercodia). Intravenous glucose tolerance testing ${ }^{12}$ was performed by infusing D-glucose $(0.1 \mathrm{~g} / \mathrm{kg}$ body weight) via rapid injection into one tail vein and collecting blood samples from the other. Multiple Comparisons were performed using the Bonferroni multiple comparisons test (GraphPad Instat 3; GraphPad, San Diego CA). Differences were considered significant if $P<0.05$ by twotailed analysis. 
A
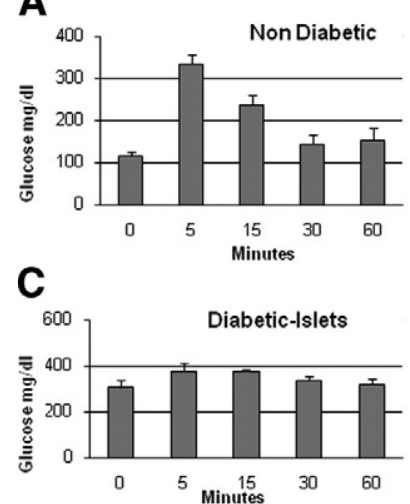

E

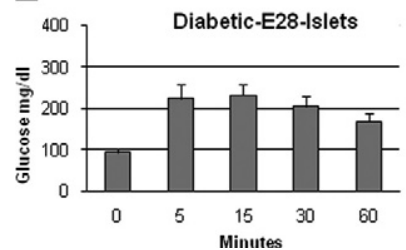

B

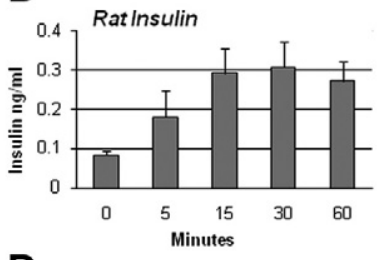

D

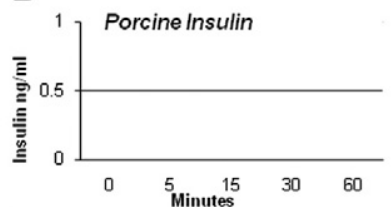

$\mathbf{F}$

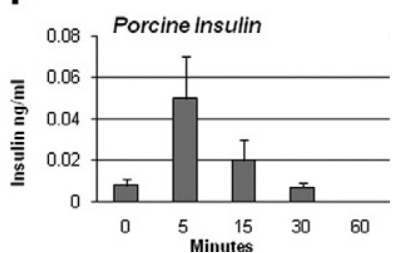

Figure 1. Levels of blood glucose $(\mathbf{A}, \mathbf{C}$, and $\mathbf{E})$ and rat insulin $(\mathbf{B})$ or porcine insulin ( $\mathbf{D}$ and $\mathbf{F}$ ) before (time 0) and following i.v. glucose administration to nondiabetic rats (A and $\mathbf{B}$ ) or STZ-diabetic rats implanted with porcine islets (C and D) or STZ-diabetic rats transplanted with E28 pig pancreatic primordia and subsequently with porcine islets $(\mathbf{E}$ and $\mathbf{F})$. Data are mean \pm SE of $n=$ 4 rats in each group.

\section{Histology}

Tissues removed from hosts were fixed in 10\% phosphate-buffered formalin. ${ }^{5-9}$ The fixative was removed, and tissues embedded in paraffin, sliced into 5 um sections and placed on glass slides in preparation for staining. Polyclonal rabbit anti-insulin serum (Accurate Chemicals, Westbury, NY) was used to detect insulin in tissue sections. Nonimmune rabbit serum was substituted for control stains. ${ }^{8}$ Sections were counterstained using hematoxylin.

\section{Detection of Insulin Transcripts Using in Situ Hybridization}

In situ hybridization was performed on sliced 5- $\mu \mathrm{m}$ paraffinembedded sliced tissue sections using a kit from GeneDetection (Bradenton, FL) exactly as before. ${ }^{8}$ Digoxinlabeled antisense probes (GeneDetection), which detect porcine, but not rat transcripts ${ }^{7}$ are complementary to nucleotides 230-264 of porcine preproinsulin cDNA: 5'GGCGGAGAACCCTCAGGCAGGTGCCGTGGAGCTGG-3' (GenBank accession number AY044828). A sense probe was used for control stains.

\section{Fluorescent in Situ Hybridization}

Fluorescence in situ hybridization in paraffin-embedded tissue sections was performed as per instructions provided by Cambio (Cambridge, U.K.). Three- to 6- $\mu \mathrm{m}$ tissue sections were dewaxed in xylene; rehydrated through graded alcohols (95; 80; 60; and 35\%) to water;

incubated with sodium thiocyanate solution (16 g dissolved in $200 \mathrm{ml}$ of water) for 10 minutes at $80^{\circ} \mathrm{C}$; washed in phosphate-buffered saline (PBS) for 10 minutes at $37^{\circ} \mathrm{C}$; incubated in a pepsin solution $(0.8 \mathrm{~g}$ of pepsin in $200 \mathrm{ml}$ of $0.1 \mathrm{M} \mathrm{HCl}$ ) for 10 minutes at $37^{\circ} \mathrm{C}$; quenched in a glycine solution [0.4 g of glycine in $200 \mathrm{ml}$ of double concentration PBS (2 mg/ml)]; washed in PBS; postfixed in a paraformaldehyde solution (8 $\mathrm{g}$ of paraformaldehyde in $200 \mathrm{ml}$ of PBS at $80^{\circ} \mathrm{C}$, cooled to room temperature before use) for 2 minutes; washed in PBS X3; dehydrated through graded alcohols; and air-dried. Prediluted Cambio X-paint mix (CA-1865-XF) was removed from the freezer and warmed to $37^{\circ} \mathrm{C}$ before $10-15 \mu$ l being applied to the center of the slide that was subsequently covered with a glass coverslip and sealed with rubber cement. The sealed slide was denatured at $80^{\circ} \mathrm{C}$ for 10 minutes and hybridized overnight in a humid chamber. The next day, coverslips were removed and the slide was washed in a formamide wash solution $[50 \mathrm{ml}$ deionized formamide mixed with $50 \mathrm{ml}$ of $2 \times$ standard saline citrate (SSC)] $37^{\circ} \mathrm{C}$ for 3 changes of 5 minutes each; washed with a stringency wash solution ( $2 \times$ standard saline citrate) at $37^{\circ} \mathrm{C}$ for 3 changes over 15 minutes; washed with a detergent wash solution $(0.1 \mathrm{ml}$ of $10 \%$ Tween 20 to $200 \mathrm{ml}$ of $4 \times$ standard saline citrate) at $37^{\circ} \mathrm{C}$ for 10 minutes; washed with PBS three times; mounted in 4',6'-Diamidino2-Phenylindole Mountant (catalog number 1124-MT-50/ 1250) and visualized using an Olympus BX61 epifluorescence microscope with software that enables generation of composite images using multichannel monochrome captures.

\section{Electron Microscopy}

Transmission electron microscopy was performed by one of us (H.L.) as before ${ }^{6}$ using paraffin embedded tissue subsequently deparaffinized in xylene and postfixed in osmium.

\section{Institutional Review Board Approval}

Animal studies were approved by the Washington University Animal Studies Committee (approval 20070288).

\section{Results}

Rats were rendered diabetic using STZ. Five days later a group into which porcine islets would be implanted 8 weeks later without prior transplantation of E28 pig pancreatic primordia (Diab-Islets) underwent sham surgery $(n=4)$. Another group was transplanted with E28 pig pancreatic primordia $(n=10)$ and subdivided into three subgroups. Eight weeks after transplantation of E28 pig pancreatic primordia: rats in one subgroup (Diab-E28E28) were transplanted with E28 pig pancreatic primordia a second time $(n=3)$; rats in a second subgroup (Diab-E28) received no further transplants $(n=3)$; rats in a third subgroup (Diab-E28-Islets) were implanted with porcine islets $(n=4)$. At 5 days after STZ administration 
Table 1. Levels of Fasting Glucose $(\mathrm{mg} / \mathrm{dl})$ in Groups of Rats

\begin{tabular}{lcccc}
\hline & Diab-E28-E28 & Diab-E28 & Diab-E28-Islets & Diab-Islets \\
\hline Before transplantation & & $384 \pm 26(n=6)$ & $359 \pm 11(n=4)$ & $378 \pm 5.0(n=4)$ \\
8 Weeks & & $232 \pm 7(n=6)$ & $213 \pm 34(n=4)$ & $309 \pm 38(n=4)$ \\
12 Weeks & $124 \pm 1(n=3)$ & $244 \pm 19(n=3)$ & $94 \pm 8(n=4)$ & $306 \pm 31(n=4)$ \\
\hline
\end{tabular}

Data are mean \pm SE.

and before any transplantation (before transplantation), fasting glucose levels in rats that were to be divided into each group averaged $>300 \mathrm{mg} / \mathrm{dl}$ and were not different, one from the other (Table 1). At 8 and 12 weeks posttransplantation of E28 pig pancreatic primordia in the Diab-E28 group, fasting glucose levels were significantly lower than before transplantation but did not differ from one another. In contrast, fasting glucose levels in the Diab-E28-E28 group measured at 12 weeks $(124 \pm 1$ $\mathrm{mg} / \mathrm{dl}$ ) were significantly lower than levels measured at 8 weeks in the Diab-E28 group and did not differ significantly from levels in a group of four nondiabetic rats $(114 \pm 9 \mathrm{mg} / \mathrm{dl})$. At 8 weeks posttransplantation of E28 pig pancreatic primordia in the Diab-E28-Islets group, fasting glucose levels were elevated relative to levels in the nondiabetic rats. However levels of glucose $(94 \pm 8$ $\mathrm{mg} / \mathrm{dl}$ ) measured at 12 weeks posttransplantation in the Diab-E28-Islets group (4 weeks after islet implantation) were not different from levels measured in the normal rats. Glucose levels at 8 in or 12 weeks from rats in the Diab-Islets group were not different from those measured before transplantation (Table 1).

Thus, as reported previously ${ }^{9}$ retransplantation of E28 pig pancreatic primordia normalizes glucose levels in rats not normalized by the first transplantation (Diab-E28E28). However, implantation of islets also normalizes glucose levels in these rats (Diab-E28-Islets). In contrast, implantation of islets in sham-operated rats does not impact on levels of fasting glucose measured 4 weeks subsequently (Diab-Islets).

Shown in Figure 1, A, C, and E, are levels of glucose and shown in Figure 1, B, D, and F, are levels of venous insulin measured using the ELISA specific for porcine insulin (Figure 1, D and F) or the ELISA that detects both rat and pig insulin (Figure 1B) each measured following administration of an i.v. glucose load to nondiabetic rats (Figure 1, A and B) or STZ-diabetic sham-operated rats 4 weeks after islet implantation (Diab-Islets) (Figure 1, C and D) or STZ-diabetic rats that had been transplanted with pig pancreatic primordia in mesentery and subsequently (4 weeks before i.v. glucose infusion) implanted with porcine islets in kidney (Diab-E28P-Islets) (Figure 1, $\mathrm{E}$ and $\mathrm{F}$ ). Fasting glucose levels in nondiabetic rats $(114 \pm 9 \mathrm{mg} / \mathrm{dll})$ and rats in the Diab-E28-Islets group (94 $\pm 8 \mathrm{mg} / \mathrm{dl})$ were lower than those in the Diab-Islets group (306 $\pm 32 \mathrm{mg} / \mathrm{dl}$ ) but not different from one another at time 0 . Levels in rats from the nondiabetic and DiabE28-Islets groups increased following i.v. glucose infusion, but 60 minutes later were not different from levels measured at time 0 . Glucose levels did not change significantly following glucose infusion in rats from the DiabIslets group. No porcine insulin $(<0.02 \mathrm{ng} / \mathrm{ml})$ was de- tected at any time in rats from the Diabetic-Islets group (Figure 1D). Rat insulin (measured using ELISA NVC 10-1137-01 in rats in which no pig tissue is present) (Figure 1B) or porcine insulin (Figure 1F), respectively, increased following the glucose infusion in nondiabetic rats and rats in the Diab-E28-Islets group. Levels of circulating porcine insulin in the Diab-E28-Islets group (Figure $1 F$ ) are lower than corresponding levels of rat insulin in nondiabetic animals (Figure 1B).

To our knowledge, recovery of native insulin secretion sufficient to normalize glucose tolerance has never been reported following administration of STZ to adult rats at doses comparable to those used in our studies ${ }^{5-8}$ which is why such animals are appropriately used to evaluate the efficacy of transplantation procedures. ${ }^{13}$ However, to provide additional evidence that the normalization of glucose tolerance shown in Figure 1E did not result from recovery of endogenous insulin production of rats in the Diab-E28-Islets group, we measured levels of rat C-peptide and compared them to levels in nondiabetic rats or a group of four diabetic rats (diabetic). Levels of rat Cpeptide in diabetic rats or rats in the Diab-E28-Islets group did not differ one from the other, were comparable to levels measured by others in STZ-diabetic rats, ${ }^{14}$ and were significantly lower than levels measured in Non Diabetic animals (Table 2). These findings are consistent with the absence of recovery of native insulin production in rats from the Diab-E28-Islets group.

Shown in Figure 2, A-D, are tissue sections of mesenteric lymph nodes from a STZ-diabetic rat into which E28 pig pancreatic primordia had been transplanted in mesentery 4 weeks previously (Diab-E28) stained using antiinsulin antibody (Figure 2, A and C), or control antiserum (Figure 2, B and D). Sections 2A and 2B are consecutive. The arrows highlight areas of in Figure 2A that are redbrown (contain insulin-positive cells) relative to comparable areas in Figure 2B. As before, in LEW rats ${ }^{5-6,8}$ or rhesus macaques ${ }^{9}$ transplanted with pig pancreatic primordia in mesentery, the insulin positive cells are located predominantly outside of germinal centers in medullary sinus (Figure 2A, arrows). Individual insulin-positive cells cannot be delineated in Figure 2A. However, individual insulin positive cells are evident in Figure $2 \mathrm{C}$ that do not stain in sections stained using control antiserum (Figure

Table 2. Levels of Fasting Rat C Peptide (pmol/L) in Groups of Rats

\begin{tabular}{ccc}
\hline Nondiabetic & Diabetic & Diab-E28-Islets \\
\hline $580 \pm 148$ & $150 \pm 53$ & $154 \pm 42$ \\
\hline
\end{tabular}

Data are mean \pm SE of $n=4$ rats in each group. 

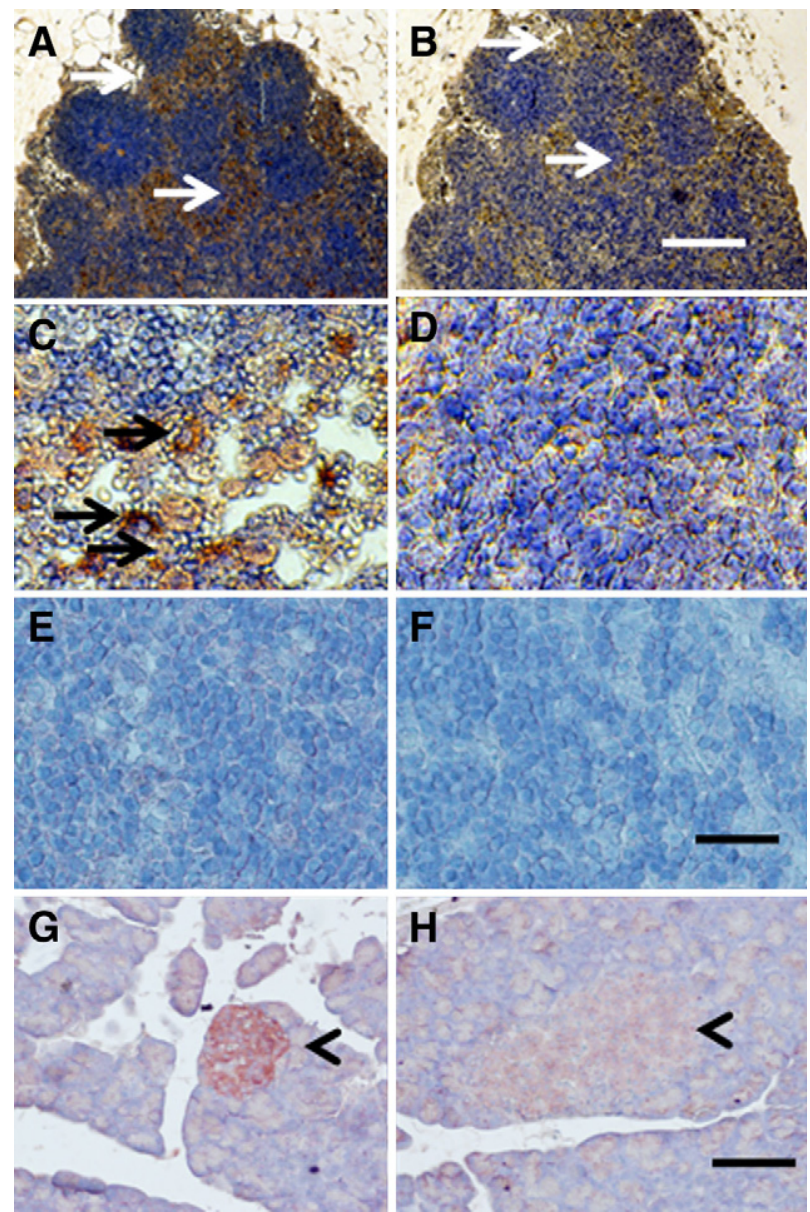

Figure 2. Photomicrographs of mesenteric lymph node (A-F) from a STZdiabetic rat into which E28 pig pancreatic primordia had been transplanted in the mesentery four weeks previously (A-D) or a nontransplanted nondiabetic rat $(\mathbf{E}$ and $\mathbf{F})$ or a pancreas from a nondiabetic nontransplanted rat $(\mathbf{G}$ and $\mathbf{H}$ ) stained using anti-insulin antibody $(\mathbf{A}, \mathbf{C}, \mathbf{E}$, and $\mathbf{G})$ or control serum $(\mathbf{B}, \mathbf{D}, \mathbf{F}$, and $\mathbf{H})$. Arrows delineate tissue that stains positive for insulin (red-brown) (A and $\mathbf{C}$ ) or negative staining tissue $(\mathbf{B})$. Arrowheads delineate islet of Langerhans $(\mathbf{G}$ and $\mathbf{H})$. Scale bars: $80 \mu \mathrm{m}(\mathbf{A}$ and $\mathbf{B}) ; 30 \mu \mathrm{m}(\mathbf{C}-\mathbf{F}) ; 100$ $\mu \mathrm{m}(\mathbf{G}$ and $\mathbf{H})$.

2D). Shown in Figure 2, E and F, are sections of mesenteric lymph nodes and in Figure 2, $\mathrm{G}$ and $\mathrm{H}$, are sections pancreas from a nontransplanted nondiabetic rat included as negative controls for lymph nodes from a transplanted rat and positive controls for insulin staining respectively. There is no insulin-positive tissue in lymph node tissue from the nondiabetic nontransplanted rat incubated with the anti-insulin antibody (Figure 2E). As would be expected, the anti-insulin antibody stains $\beta$ cells in an islet of Langerhans in the pancreas from the non-diabetic non-transplanted rat (arrowhead; Figure $2 \mathrm{G}$ ). Islet tissue does not stain if control antiserum is substituted (arrowhead; Figure $2 \mathrm{H}$ ).

Figure 3 illustrates sections of mesenteric lymph node from a formerly diabetic rat following transplantation of embryonic pancreas in mesentery followed by implantation of pig islets in kidney (Diab-E28-Islets). Figure 3, A and $\mathrm{B}$, is consecutive, and the arrows highlight comparable areas. Cells in medullary sinus expressing insulin stain with use of the anti-insulin antibody (Figure 3, A and C) but not the control antiserum (Figure 3, B and D). As
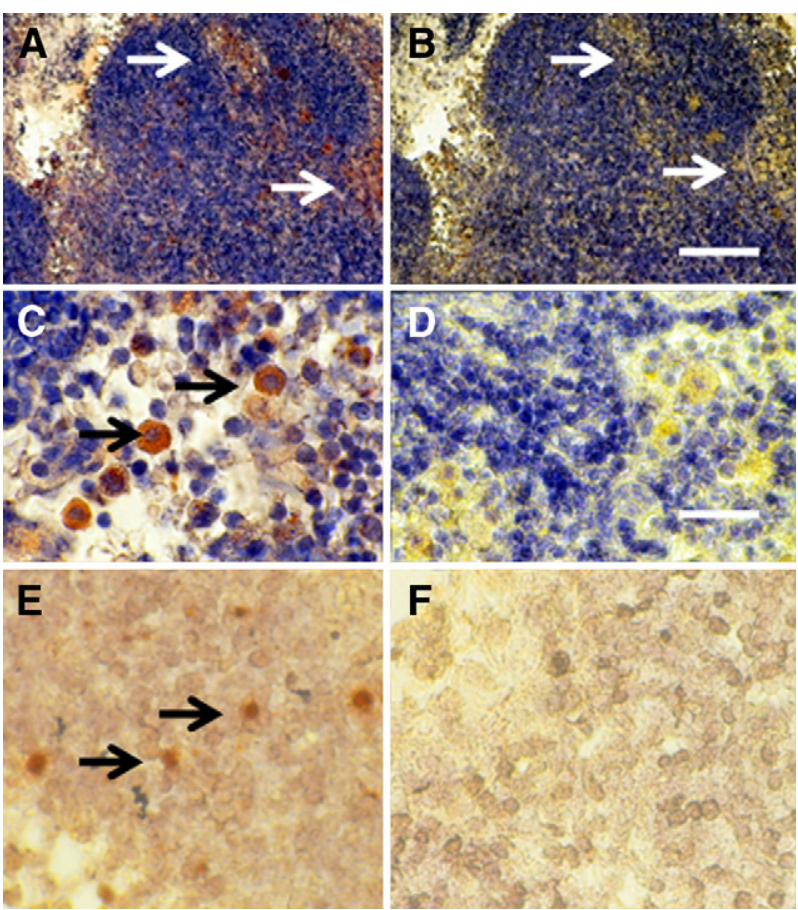

Figure 3. Photomicrographs of mesenteric lymph node from a formerly diabetic rat into which E28 pig pancreatic primordia and pig islets had been transplanted in the mesentery and kidney respectively stained using antiinsulin antibody (A and $\mathbf{C}$ ) or control antibody (B and $\mathbf{D}$ ) and sections hybridized to antisense (E) or sense (F) porcine proinsulin mRNA probes. Arrows delineate tissue that stains positive for insulin (red-brown) (A and $\mathbf{C}$ ) or negative staining tissue (B) or positive staining for porcine proinsulin mRNA (E). Scale bars: $80 \mu \mathrm{m}(\mathbf{A}$ and $\mathbf{B})$ and $25 \mu \mathrm{m}(\mathbf{C}-\mathbf{F})$.

for Figure 2A, individual insulin-positive cells cannot be delineated in Figure 3A. However, individual insulin positive cells are evident in Figure 3C. Also shown in Figure 3 are sections incubated with antisense (Figure $3 \mathrm{E}$ ) or sense (Figure 3F) porcine proinsulin mRNA probes. As before, ${ }^{7,8}$ hybridization occurs in cells incubated with the antisense but not with the sense probe.

Figure 4 is a photograph of a kidney from a STZtreated rat that had been transplanted with embryonic pig pancreas in mesentery, taken 4 weeks after implantation of islets in kidney. As previously described by others in rat kidney following islet isotransplantation ${ }^{15}$ a distinct, whitish well-demarcated graft, which can be easily distinguished from the surrounding renal parenchyma is observed (white arrow) along with large intracapsular venous blood vessels that radiate from the graft out into the renal parenchyma (black arrow).

Figure 5 shows sections from a kidney from a STZdiabetic rat transplanted previously with embryonic pig pancreas in mesentery and subsequently with pig islets in kidney obtained 4 weeks after transplantation of islets. Sections are stained using anti-insulin antibodies (Figure 5, A and C) or control serum (Figure 5, B and D). As would be expected for kidney that filters, reabsorbs, and secretes insulin, ${ }^{16}$ proximal tubules $(P T)$ in Figures $5 \mathrm{~A}$ are positive (red brown) relative to comparable structures in Figure 5B. Cells that stain for insulin (Figure 5A), but not with control serum (Figure $5 \mathrm{~B}$ ) are present in an expanded subcapsular space (Figure 5, A and B, arrow- 


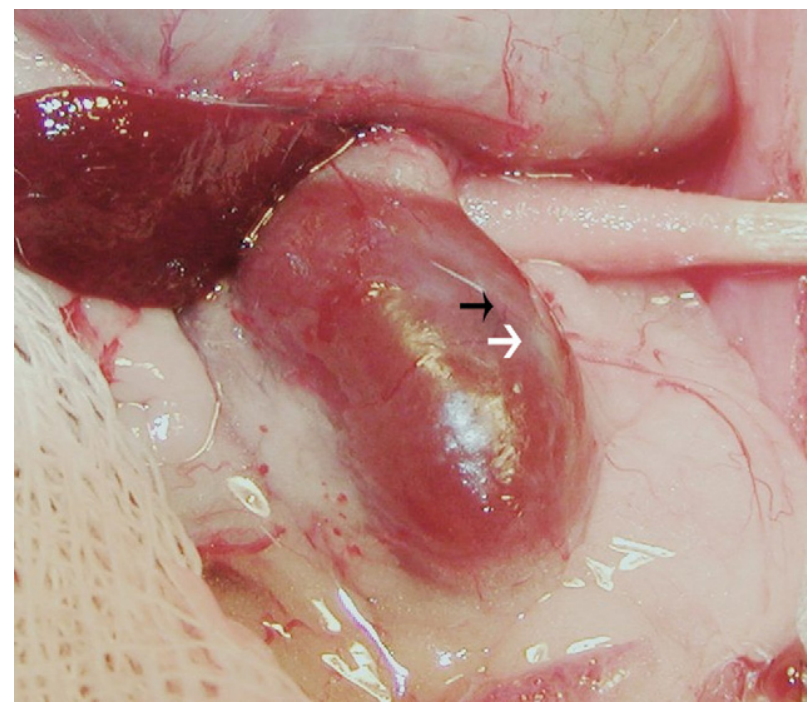

Figure 4. Photograph of kidney from a STZ-treated rat transplanted previously with embryonic pig pancreas in mesentery. The photo was taken four weeks after implantation of pig islets in kidney. The whitish well-demarcated graft (white arrow) and the origin of a venous blood vessel (black arrow) are shown.

heads). Figure 5C shows a higher magnification of the subcapsular space. The cells that stain positive for insulin (red-brown stain) are polygonal with round nuclei and abundant cytoplasm (arrow), a $\beta$ cell morphology. ${ }^{5-8}$ Also shown in Figure 5 are sections incubated with antisense (Figure 5E) or sense (Figure 5F) porcine proinsulin mRNA probes. Hybridization occurs with cells hybridized to the former (arrows) but not the latter probe.

The contralateral (nontransplanted) kidney from a STZdiabetic rat transplanted previously with embryonic pig pancreas in mesentery and subsequently with pig islets in the ipsilateral kidney is shown in Figure 6, A-D. Sections obtained 4 weeks after islet transplantation are stained using anti-insulin antibodies (Figure 6, A and C) or control serum (Figure 6, B and D). A low magnification view of the contralateral kidney shows normal renal morphology with no evidence of engrafted tissue (Figure 6, A and $\mathrm{B}$ ). As in Figure 5, PT in Figure 6A are positive (red brown) relative to comparable structures in Figure 6B. Immunoreactive insulin (non-reabsorbed) ${ }^{16}$ is also evident in the medulla $(M)$ shown in Figure $6 \mathrm{~A}$ relative to Figure $6 \mathrm{~B}$. In contrast to what is observed in the transplanted kidney (Figure 5, A and B), there is no expansion of the subcapsular space in the contralateral kidney (Figure 6, A and C, arrowheads) and no cells are present with $\beta$ cell morphology as shown in Figure 5, C. Figure 6, E and F, shows the subcapsular space of a kidney from a rat implanted with porcine islets 4 weeks previously with no prior transplantation of E28 pig pancreatic primordia (Diab-Islets). Sections are stained using anti-insulin antibodies (Figure 6E) or control serum (Figure 6F). Insulin staining of $P T$ is evident in Figure 6E. The subcapsular space (Figure 6E, arrowheads) is expanded relative to that depicted in Figure 6, A and $\mathrm{C}$. However, there are no cells with $\beta$ cell morphology that stain for insulin (Figure 6E).

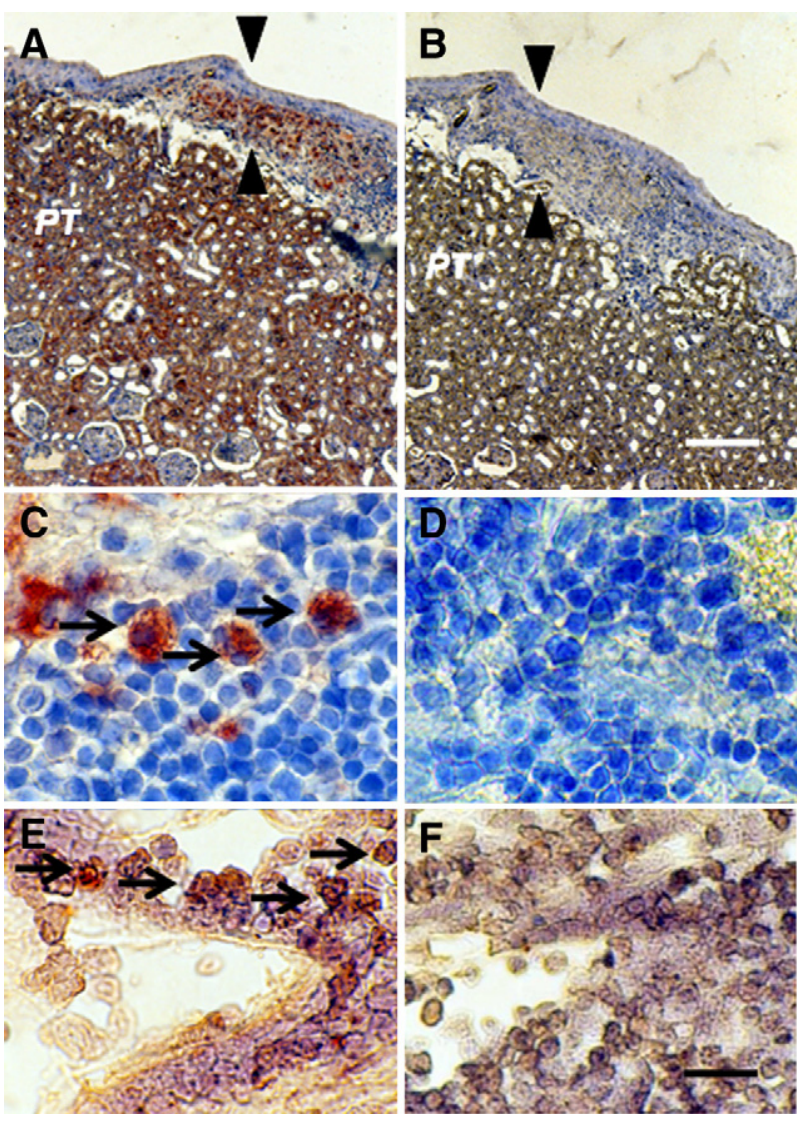

Figure 5. Photomicrographs of kidney from a diabetic rat into which embryonic pig pancreas had been transplanted in mesentery and pig islets had been transplanted subsequently in kidney stained using anti-insulin antibody (A and $\mathbf{C}$ ) or control antibody ( $\mathbf{B}$ and $\mathbf{D}$ ) and sections hybridized to antisense (E) or sense (F) porcine proinsulin mRNA probes. Arrowheads delineate an expanded subcapsular space (A and $\mathbf{B})$. Arrows delineate tissue in the subcapsular space that stains positive for insulin (red-brown) (C) or positive staining for porcine proinsulin mRNA (E). PT, A and B. Scale bars 80 $\mu \mathrm{m}(\mathbf{A}$ and $\mathbf{B})$ and $10 \mu \mathrm{m}(\mathbf{C}-\mathbf{F})$

To provide additional evidence that pig cells are present in kidneys and mesenteric lymph nodes of rats transplanted with pig pancreatic primordia and subsequently with porcine islets, we performed fluorescent in situ hybridization using a probe specific for the pig $X$ chromosome. Shown in Figure 7A (arrows) are pig $X$ chromosomes in nuclei of cells from a normal porcine pancreas (positive control). Figures 7, B and C (arrows), show, respectively, in tissue obtained 4 weeks after islet transplantation: pig X chromosomes in the nuclei of cells in the renal subcapsular space [between a tubule $(T)$ and the renal capsule membrane $(R C)]$; and pig $X$ chromosomes in the nuclei of cells outside of a germinal center (GC) of a mesenteric lymph node. There are no cells containing pig $X$ chromosomes in renal cortex from the transplanted kidney (Figure 7D), consistent with the subcapsular localization of the insulin positive and porcine proinsulin mRNA containing cells (Figure 5) and with species specificity of the pig $X$ chromosome probe (host rats are females).

We have shown previously ${ }^{6}$ using electron microscopy that cells with $\beta$ cell morphology containing granules, some of which have a crystalline core surrounded by a 


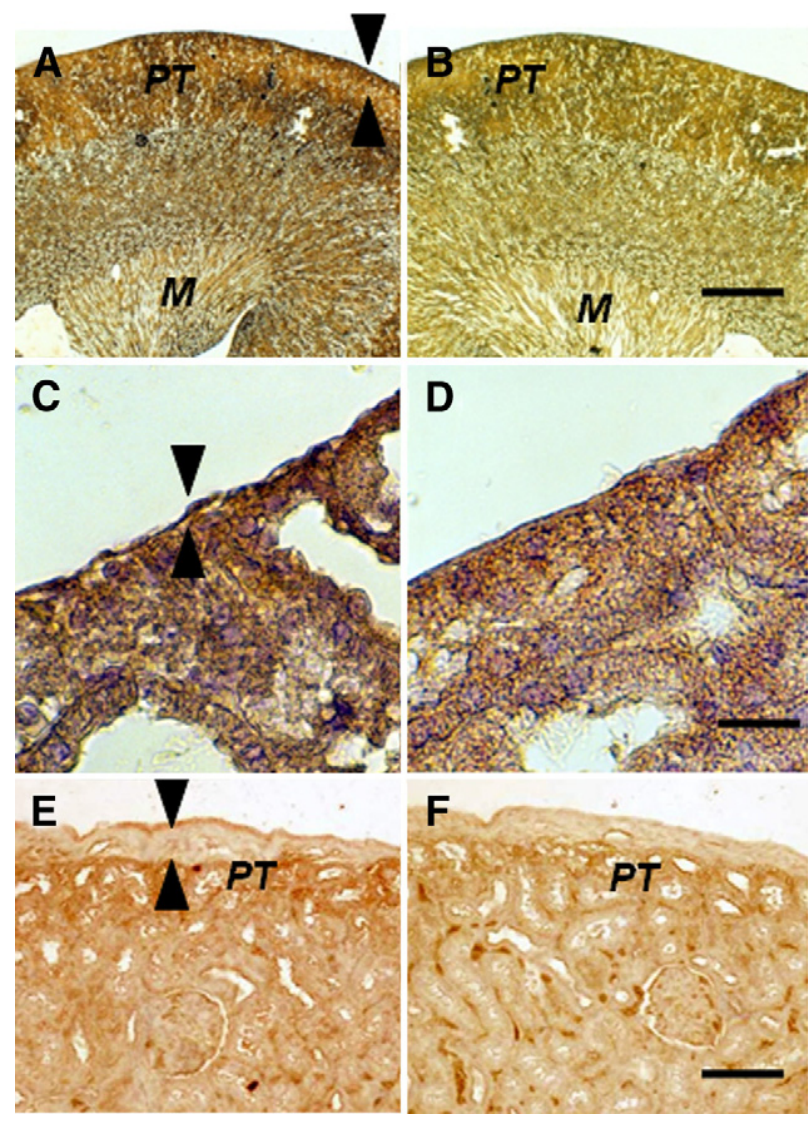

Figure 6. Photomicrographs of the contralateral kidney from a diabetic rat into which embryonic pig pancreas had been transplanted in the mesentery and pig islets had been implanted subsequently in the other kidney (A-D) or of a kidney from a diabetic rat in which pig islets had been implanted without prior transplantation of E28 pig pancreatic primordia (E and $\mathbf{F})$ stained using anti-insulin antibody $(\mathbf{A}, \mathbf{C}$, and $\mathbf{E})$ or control antiserum $(\mathbf{B}, \mathbf{D}$, and $\mathbf{F}$ ). Arrowheads delineate a normal sized subcapsular space $(\mathbf{A}, \mathbf{C})$ or expanded subcapsular space (E). PT, A, B, E, and F. $M$, medulla (A and B). Scale bars: $100 \mu \mathrm{m}(\mathbf{A}$ and $\mathbf{B}), 10 \mu \mathrm{m}(\mathbf{C}$ and $\mathbf{D})$, or $40 \mu \mathrm{m}(\mathbf{E}$ and $\mathbf{F})$.

clear space, are present in mesentery of rats following transplantation of E28 pig pancreatic primordia. To ascertain whether similar cells are present in kidneys of rats transplanted with E28 pig pancreatic primordia and subsequently with porcine islets, we performed electron microscopy on tissue obtained 4 weeks after islet transplantation. Shown in Figure 8A (arrow) is a cell in the renal subcapsular space with $\beta$ cell morphology [containing granules $(250-400 \mathrm{~nm})$ which have a crystalline core

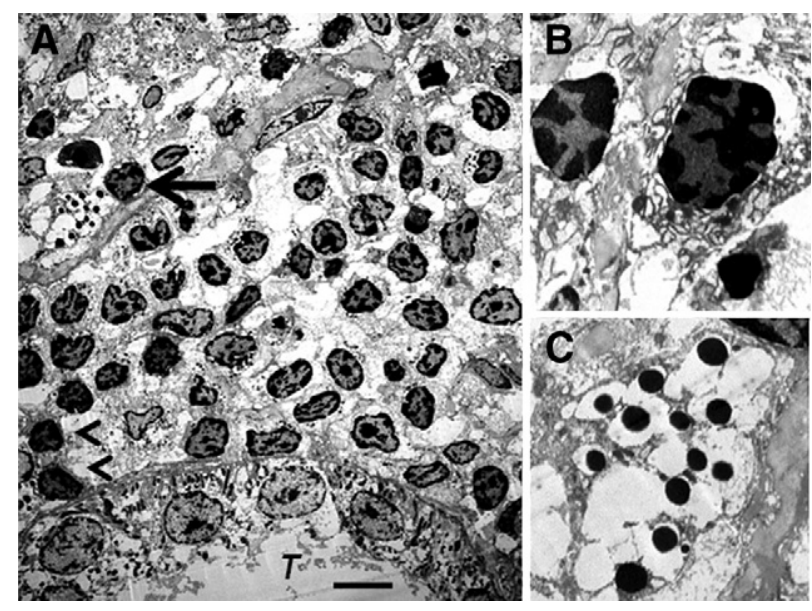

Figure 8. Electron micrographs of rat kidney following sequential transplantation of E28 pig pancreatic primordia in mesentery and implantation of porcine islets in kidney. A, Subcapsular space. T, renal tubule. Cell containing granules with a crystalline core surrounded by a clear space is delineated by an arrow; macrophages are delineated by arrowheads. B, Enlargement of macrophages. C, Enlargement of granules with a crystalline core surrounded by a clear space. Scale bar: $5 \mu \mathrm{m}(\mathbf{A})$.

surrounded by a clear space]. A renal tubule $(T)$ is labeled. A mononuclear cell infiltrate in the renal subcapsular space consists predominantly of macrophages. Two are labeled (arrowheads). Figure 8B is a high-power view of two others. Figure $8 \mathrm{C}$ shows a high power view of the granules with a crystalline core surrounded by a clear space.

Figure 9 shows levels of fasting glucose measured over time in STZ diabetic rats transplanted with E28 pig pancreatic primordia (E28) after measurements on week 1 and implanted with porcine islets (islets) after measurements on week 8 (Diab-E28-Islets group). Glucose levels were reduced to normal on week 12 (4 weeks following transplantation of islets) and remained so for 16 weeks thereafter (week 28).

Figure 10 shows sections from a kidney of one of the rats from the Diab-E28-Islets group euthanized on week 28 (20 weeks following islet implantation).Sections are stained using anti-insulin antibodies (Figure 10A) or control serum (Figure 10B). Cells that stain for insulin (Figure $10 \mathrm{~A}$, arrows) but not with control serum (Figure 10B, arrows) are present in an expanded subcapsular space. The cells that stain positive for insulin (red-brown stain)
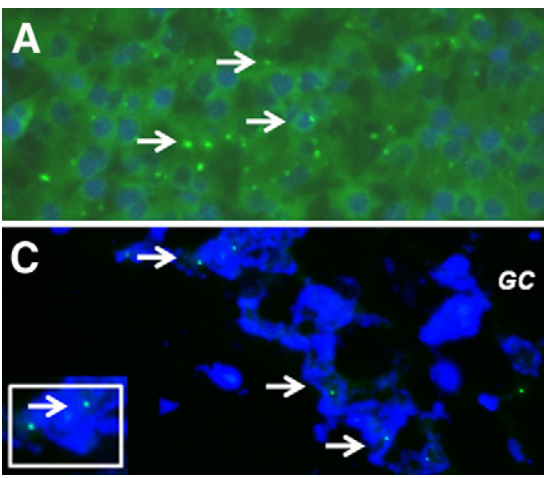
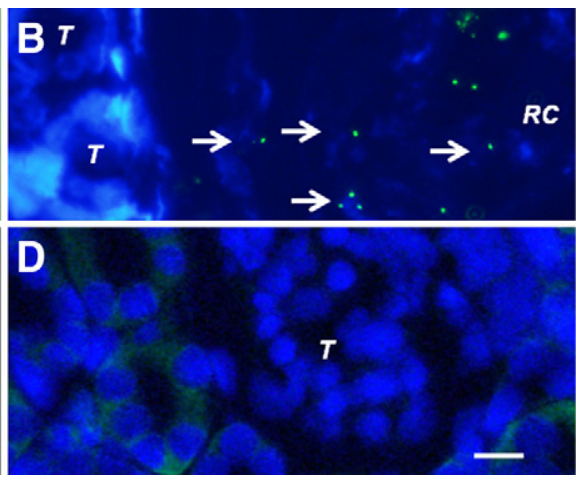

Figure 7. Fluorescence microscopy of tissue sections originating from a normal porcine pancreas (A) or a diabetic rat that had been transplanted with embryonic pig pancreas $(\mathbf{B}-\mathbf{D})$ in mesentery and subsequently with porcine islets in kidney: B, a subcapsular section from kidney; $T$, tubule, $R C$, renal capsule; $\mathbf{C}$, a section of mesenteric lymph node, GC, germinal center, inset enlargement; and $\mathbf{D}$, renal cortex, $T$, tubule. Arrows (A-C) delineate pig X chromosomes. Scale bar: $10 \mu \mathrm{m}$ (D). 


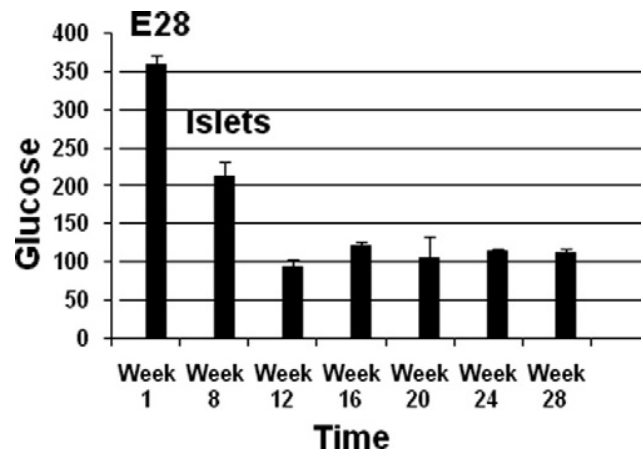

Figure 9. Levels of fasting glucose measured over time in STZ diabetic rats transplanted with E28 pig pancreatic primordia (E28) after measurements on week 1 and implanted with porcine islets (islets) after measurements on week 8. Data are mean \pm SE of $n=4$ rats (weeks 1-12) or $n=2$ rats (weeks 16-28)

are polygonal with round nuclei and abundant cytoplasm (arrow), a $\beta$ cell morphology. ${ }^{5-8}$

\section{Discussion}

STZ is a glucose-conjugated nitrosourea which is believed to gain access to $\beta$ cells by the mechanisms that sense the blood glucose level. ${ }^{17}$ It is used widely to induce diabetes in animals $2,5,6,8,9,12-14,17-24$ and is particularly appropriate for islet transplantation because insulin is supplied only from the graft islet cells. ${ }^{13}$ STZ produces a rapid cell necrosis. If given in sufficiently large amounts to a rat $(40-100 \mathrm{mg} / \mathrm{kg}),{ }^{24}$ a single dose will destroy most of the $\beta$ cells within the pancreas and induce a permanent severe diabetes. A lower dose will kill fewer cells and provoke a less severe diabetes. ${ }^{17}$ The diabetogenic action of the same dose of STZ varies from animal to animal. However, neither severe STZ-induced diabetes nor the less severe form is reversible in the rat. ${ }^{13,17,18}$ Previously ${ }^{25}$ we have performed immunohistochemistry for insulin in pancreas from LEW rats treated with STZ at doses used in the present study, glucose levels in which had been normalized by transplantation of E28 pig pancreatic primordia. We compared pancreas histology in STZ-treated rats to age-matched nondiabetic LEW animals. As is characteristic for STZ diabetes in the
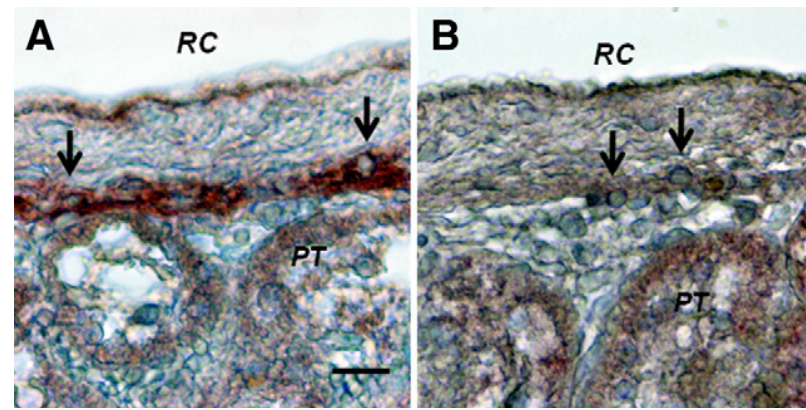

Figure 10. Photomicrographs of kidney from a diabetic rat into which embryonic pig pancreas had been transplanted in mesentery and pig islets had been transplanted subsequently in kidney stained using anti-insulin antibody (A) or control antibody (B). Tissue was obtained 20 weeks after islet transplantation. Arrows delineate cells in the subcapsular space with $\beta$ cell morphology. $R C$, renal capsule; $P T, \mathbf{A}$ and $\mathbf{B}$. Scale bar: $10 \mu \mathrm{m}(\mathbf{A})$. rat, ${ }^{18}$ we demonstrated a depression in the number and volume of $\beta$ cells within islets in from animals that received STZ and a substantial reduction in islet volume relative to the age-matched nondiabetic LEW rats, consistent with the marked reduction, but not elimination of pancreatic insulin in rats after STZ administration. ${ }^{24}$

Transplantation of embryonic pancreas to replace the function of diseased organs offers theoretical advantages relative to transplantation of either pluripotent ES cells or of fully differentiated (adult) pancreas or islets $^{5-9,19,22,26-29}$ : i) Unlike ES cells, pancreatic primordia differentiate along defined organ-committed lines. There is no requirement to steer differentiation and no risk of teratoma formation. ${ }^{26}$ In the case of embryonic pancreas, the glucose sensing and insulin releasing functions of $\beta$ cells that differentiate from primordia are functionally linked. ${ }^{5-9,12}$ ii) The growth potential of cells within embryonic pancreas is enhanced relative to those in terminally-differentiated pancreas or islets. ${ }^{5-9,12,22,26-28}$ iii) The cellular immune response to transplanted primordia obtained early during embryogenesis is attenuated ${ }^{5-9,22,26-28}$ relative to that directed against adult islets $^{2-4,20,21,23,30-34}$. iv) Early organ primordia are avascular. The ability of cellular primordia to attract a host vasculature renders them less susceptible to humoral rejection than is adult pancreas with donor blood vessels transplanted across a discordant xenogeneic barrier. ${ }^{22,26-28}$ v) Organ primordia differentiate selectively. In the case of E28 embryonic pig pancreas, exocrine pancreatic tissue does not differentiate following transplantation, obviating complications that can result from exocrine components such as the enzymatic autodigestion of host tissues. ${ }^{5-9,12}$

While glucose tolerance can be normalized in nonimmune-suppressed diabetic rats by transplantation of E28 pig pancreatic primordia, ${ }^{5-8}$ it has proven to be more difficult to normalize glucose tolerance in nonimmune-suppressed rhesus macaques. ${ }^{9}$ Exogenous insulin requirements are reduced in transplanted macaques. However, glucose tolerance in the macaques remains abnormal even after multiple transplantation surgeries. ${ }^{9}$ The most likely explanation is that the macaques we used for experiments weigh $\sim 20$ times as much as the rats. ${ }^{5,9} \mathrm{~A}$ STZ-diabetic rat can be rendered normoglycemic by transplantation of five pig pancreatic primordia. ${ }^{7,8}$ Extrapolating, it would take 100 primordia to normalize glucose tolerance in a rhesus macaque. This would require the sacrifice of about seven pregnant sows and would require a good deal of surgical time with the attendant complications. In lieu of increasing the number of transplanted primordia to normalize glucose tolerance in diabetic rhesus macaques, we embarked on a series of experiments to determine whether porcine islets, a more easily obtainable and possibly more robust source of insulin-producing cells, could be substituted in animals rendered tolerant to E28 pig pancreatic primordia. Our first step, reported here, was to determine using rats, ${ }^{5-8}$ whether previous transplantation of the primordia renders hosts tolerant to islets.

To our knowledge, ours is the first report describing sustained normalization of glucose tolerance following transplantation of porcine islets in nonimmune sup- 
pressed, immune competent rodents. Levels of fasting glucose in the STZ-diabetic rats are normalized following transplantation of E28 pig pancreatic primordia followed by implantation of islets (Diab-E28-Islets) (Table 1). Normalization of glucose tolerance in the Diab-E28-Islets group (Figure 1E) cannot be attributed to the first transplantation of E28 pig pancreatic primordia alone, since rats in the Diab-E28 group (without a second transplant) remain hyperglycemic at 12 weeks (Table 1). Lanza et al transplanted 10,000-20,000 adult porcine islets equivalents in the peritoneum of three nonimmune-suppressed STZ-diabetic LEW rats included as a control group for macroencapsulated islets. ${ }^{20}$ Fasting glucose levels fell from $\sim 600$ to $300 \mathrm{mg} / \mathrm{dl} 1$ day after transplantation, but returned to baseline (elevated) levels within 1 week. Wennberg et $\mathrm{al}^{23}$ transplanted adult porcine islets $(20,000$ 30,000 islets equivalents) beneath the kidney capsule of nonimmune-suppressed STZ-diabetic LEW rats included in a control group. Normoglycemia was sustained for $5.5 \pm 0.3$ days following which blood glucose levels returned to diabetic levels. ${ }^{23} \mathrm{Wu}$ et $\mathrm{al}^{21}$ transplanted 4000-5000 islets equivalents beneath the renal capsule of diabetic C57BL/6 mice. Levels of blood glucose fell transiently, but returned to diabetic levels within a week.

Here we document using immune histochemistry for insulin (Figures 5 and 10), in situ hybridization for porcine proinsulin mRNA (Figure 5), and fluorescent in situ hybridization for the pig $X$ chromosome (Figure 7) that pig cells engraft in kidney following transplantation of E28 pig pancreatic primordia in mesentery and subsequent implantation of porcine islets in kidney. Pig cells do not engraft in host kidney following implantation of islets without prior transplantation of E28 pig pancreatic primordia (Figure 6, E and F) or following transplantation of E28 pig pancreatic primordia without subsequent implantation of islets $^{5-8}$ or in the contralateral kidney (Figure 6, A-D). Thus, the cells illustrated in Figures 5, 7, and 10 are almost certainly derived from the porcine islets and not from the embryonic pancreas transplants. To our knowledge, ours is the first report describing sustained survival of $\beta$ cells following transplantation of porcine islets beneath the renal capsule of nonimmune suppressed immune sufficient rodents. In previous studies that did not employ transplantation of E28 pig pancreatic primordia before implantation of islets or islet cell clusters ${ }^{21,23,30}$ complete destruction of grafts was evident within 2 weeks. Preimmunization of rats by subcutaneous injection of porcine islet cell clusters accelerates the rejection of islet clusters transplanted subsequently beneath the renal capsule. ${ }^{30}$ In contrast, prior transplantation of E28 pig pancreatic primordia in mesentery enables survival of an insulin-expressing cell component of porcine islets implanted subsequently in rat kidneys (Figures 5 and 10).

Levels of circulating porcine insulin following i.v. glucose infusion in rats from the Diab-E28-Islets group (Figure $1 \mathrm{~F})$ are lower than levels of circulating rat insulin following IV glucose infusion in nondiabetic animals (Figure 1B). This could reflect an increased potency of porcine insulin in rats relative to rat insulin. Alternatively, it could reflect ectopic insulin secretion in response to i.v. glucose in rats with E28 pig pancreatic primordia trans- planted in mesentery and porcine islets implanted in kidney comparable to ectopic insulin secretion following islet transplantation in humans. Intravenous glucose tolerance can be normalized in immune suppressed humans following islet allotransplantation. However, insulin secretion after glucose infusion (area under the curve) is severalfold lower than in nondiabetic humans. ${ }^{35}$ Ryan et $\mathrm{al}^{35}$ attribute this to inherent denervation of transplanted islets and the absence of specialized native islet vasculature. Similar differences between enervation and vascularization in transplants and native islets could explain differences between insulin release shown in Figure 1, B and $F$, in the setting of comparable glucose tolerance (Figure 1, A and E).

Korsgren ${ }^{34}$ has proposed that rejection of an islet cell xenograft is dependent on two different cellular mechanisms. The first is recognition of pig MHC molecules by cytotoxic lymphocytes via both direct and indirect pathways of antigen recognition, as occurs following rejection of an allograft. The second is an immune response characterized by $T$ cell dependent infiltration of macrophages with histopathological characteristics of delayed-type hypersensitivity. In nonimmune-suppressed rodents transplanted with porcine islets, the second mechanism predominates. In nonimmune-suppressed nonhuman primates, rejection is dominated by the first. However, the first process is inhibited in immune suppressed nonhuman primates and the second, less sensitive to immune suppression, is revealed. ${ }^{34}$

The presence of macrophages beneath the renal capsule of rats transplanted with porcine islets subsequent to E28 pig pancreatic primordia (Figure 8) may reflect the second cellular mechanism described by Korsgren ${ }^{34}$ in the context of tolerance to a cell component of porcine islets to which rats have been rendered tolerant by prior transplantation of E28 pig pancreatic primordia. Alternatively, because porcine islets are rejected by immune competent rats without prior transplantation of E28 pig pancreatic primordia (Figure 6, E and F), the macrophages may represent a population of M2 phenotype partially responsible for the tolerance we observe. ${ }^{36}$

Schroeder et $\mathrm{al}^{37}$ define transplantation tolerance as immune unresponsiveness to the transplanted organ, but not to other antigens, in the absence of ongoing immunosuppression. LEW rats transplanted with E28 pig pancreatic primordia retain reactivity to other porcine xenoantigens $\left[(\text { E28 pig renal primodia are rejected })^{6}\right]$. Thus, our findings are consistent with induction of specific tolerance $^{37}$ to a cell component of adult porcine islets implanted in LEW rats (cells that express insulin) by previous transplantation of E28 pig pancreatic primordia. Given similarities in the host immune responses in rodents and nonhuman primates to porcine xenografts, ${ }^{30,32}$ studies are warranted using the latter transplanted with E28 pig pancreatic primordia as hosts for porcine islets.

Though not observed following xenotransplantation under all conditions, ${ }^{28}$ host tolerance to early stage pancreatic progenitors has been reported twice previously. Eloy et $\mathrm{al}^{19}$ described normalization of glucose posttransplantation of E15 but not E18 embryonic chick pancreas into nonimmmune suppressed STZ-diabetic immune 
competent rats. Abraham et $\mathrm{al}^{38}$ described successful xenoengraftment in multiple organs of human pancreatic islet-derived progenitor cells infused in nonimmunosuppressed immune-competent mice. Neither the present studies nor prior work $^{5-9,19,38}$ defines a mechanism for the tolerance. However, it is possible that transplantation of embryonic pancreas obtained early during differentiation $^{5-9,19}$ is particularly suited to its induction.

Type 1 diabetes is an autoimmune disease and autoimmunity persists even after complete $\beta$ cell destruction. ${ }^{4}$ Owing to the primed immune system and persistent autoimmunity that exists in type 1 diabetes, there might still be a need for immune suppressing drugs to permit engraftment of E28 pig pancreatic primordia and porcine islets following transplantation in type 1 diabetics.

\section{Acknowledgment}

We thank Nicholas Benshoff for the technical assistance of preparation of porcine islets for this study.

\section{References}

1. Groth CG, Korsgren O, Tibell A, Tollemar J, Moller E, Bolinder J, Ostman J, Reinholt FR, Hellerstrom C, Andersson A: Transplantation of porcine fetal pancreas to diabetic patients. Lancet 1994, 344:1402-1404

2. Hering B, Wijkstrom M, Graham M, Hardstedt M, Aasheim TC, Jie T, Ansite JD, Nakano M, Cheng J, Li W, Moran K, Christians U, Finnegan C, Mills CD, Sutherland DE, Bansal-Pakala P, Murtaugh MP, Kirchhof $\mathrm{N}$, Schuurman HJ: Prolonged diabetes reversal after intraportal xenotransplantation of wild-type porcine islets in immunosuppressed nonhuman primates Nat Med 2006, 12:301-303

3. Cardona K, Korbutt GS, Milas Z, Lyon J, Cano J, Jiang W, BelloLahorn H, Hacquoil B, Strobert E, Gangappa S, Weber CJ, Pearson TC, Rajotte RV, Larsen CP: Long-term survival of neonatal porcine islets in non-human primates by targeting costimulation pathways. Nat Med 2006, 12:304-306

4. Hering BJ, Walawalkar N: Pig-to-nonhuman primate islet xenotransplantation. Transplant Immunol 2009, 21:81-86

5. Rogers SA, Chen F, Talcott M, Hammerman MR: Islet cell engraftment and control of diabetes in rats following transplantation of pig pancreatic anlagen. Am J Physiol 2004, 286:E502-E509

6. Rogers SA, Liapis H, Hammerman MR: Normalization of glucose post-transplantation of pig pancreatic anlagen into non-immunosuppressed diabetic rats depends on obtaining anlagen prior to embryonic day 35. Transplant Immunol 2005, 14:67-75

7. Rogers SA, Chen F, Talcott M, Liapis H, Hammerman MR: Glucose tolerance normalization following transplantation of pig pancreatic primordia into non-immunosuppressed diabetic ZDF rats. Transplant Immunol 2006, 16:176-184

8. Rogers SA, Hammerman MR: Normalization of glucose post-transplantation into diabetic rats of pig pancreatic primordia preserved in vitro. Organogenesis 2008, 4:48-51

9. Rogers SA, Chen F, Talcott MR, Faulkner C, Thomas JM, Thevis M, Hammerman MR: Long-term engraftment following transplantation of pig pancreatic primordia into non-immunosuppressed diabetic rhesus macaques Xenotransplantation 2007, 14:591-602

10. Swanson CJ, Olack BJ, Goodnight D, Zhang L, Mohanakumar T: Improved methods for the isolation and purification of porcine islets. Hum Immunol 2001, 62:739-749

11. Rogers SA, Lowell JA, Hammerman NA, Hammerman MR: Transplantation of developing metanephroi into adult rats. Kidney Int 1998, 54:27-37

12. Rogers $S A$, Liapis $H$, Hammerman MR: Intraperitoneal transplantation of pancreatic anlagen. ASAIO J 2003, 49: 527-532

13. Hamamoto Y, Tsuura Y, Fujimoto S, Nagata M, Takeda T, Mukai E,
Fujita J, Yamada Y, Seino Y: Recovery of function and mass of endogenous $\beta$ cells in streptozotocin-induced diabetic rats treated with islet transplantation. Biochem Biophys Res Commun 2001, 287:104-109

14. Cotter MA, Ekberg K, Wahren J, Cameron NE: Effects of proinsulin C-Peptide in experimental diabetic neuropathy vascular actions and modulation by nitric oxide synthase inhibition. Diabetes 2003, 52:1812-1817

15. Kallskog O, Kampf C, Andersson A, Carlsson PO, Hansell P, Johansson $\mathrm{M}$, Jansson L: Lymphatic vessels in pancreatic islets implanted under the renal capsule in rats. Am J Transplant 2006, 6:680-686

16. Hammerman MR: The interaction of insulin with the renal proximal tubular cell. Am J Physiol 1985, 249:F1-F11

17. Slack JMW: Developmental biology of the pancreas. Development 1995, 121:1569-1580

18. Chang AY, Dinai AR: Chemically and hormonally induced diabetes mellitus. The diabetic pancreas. Edited by BW Volk and ER Arquilla. New York, Plenum Press, 1985, pp 415-438

19. Eloy R, Haffen K, Kedinger M, Griener JF: Chick embryo pancreatic transplants reverse experimental diabetes of rats. J Clin Invest 1979, 64:361-373

20. Lanza RL, Butler DH, Borland KM, Staruk JE, Faustman DL, Solomon BA, Muller TE, Rupp RG, Monaco AP, Chick L: Xenotransplantation of canine, bovine, and porcine islets in diabetic rats without immunosuppression. Proc Natl Acad Sci USA 1991, 88:11100-11104

21. Wu GS, Korsgren O, Zhang JG, Song ZS, Van Rooijen N, Tibell A: Pig islet xenograft rejection is markedly delayed in macrophage-depleted mice: a study in streptozotocin diabetic animals. Xenotransplantation 2000, 7:214-220

22. Hecht G, Eventov-Friedman S, Rosen C, Shezen E, Tchorsh D, Aronovich A, Freud E, Golan H, El-Hasid R, Katchman H, Hering BJ, Zung A, Kra-Oz Z, Shaked-Mishnan P, Yusin A, Idelevich P, Tobar A, Harmelin A, Bachar-Lustig E, Reisner Y: Embryonic pig pancreatic tissue for the treatment of diabetes in a nonhuman primate model. Proc Natl Acad Sci USA 2009, 106:8659-8664

23. Wennberg L, Song Z, Benet W, Zhang J, Nava S, Sundberg B, Bari S Groth CG, Korsgren O: Diabetic rats transplanted with adult porcine islets and immunosuppressed with cyclosporine A mycophenolate mofetil and leflunomide remain normoglycemic for up to 100 days. Transplantation 2001, 71:1024-1033

24. Junod A, Lambert AE, Staffacher W, Renold AE: Diabetic action of streptozotocin: relationship of dose to metabolic response. J Clin Invest 1969, 48:2129-2139

25. Hammerman MR: Growing new endocrine pancreas in situ. 2006. Clin. Exp Nephrol 10:1-7

26. Eventov-Friedman $\mathrm{S}$, Katchman $\mathrm{H}$, Shezen E, Arnivich A, Tchorsh D, Dekel B, Freud E, Reisner Y: Embryonic pig liver, pancreas and lung as a source for transplantation: optimal organogenesis without teratoma depends on distinct time windows. Proc Natl Acad Sci USA 2005, 102:2928-2933

27. Eventov-Friedman S, Tchorsh D, Katchman H, Shezan E, Arnovich A Hecht G, Dekel B, Rechavi G, Blazar B, Feine, Tal O, Freud E, Reisner $Y$ : Embryonic pig pancreatic tissue transplantation for the treatment of diabetes. PLoS Med 2006, 7:1165-1177

28. Tchorsh-Yutsis D, Hecht G, Aronovich A, Shezen E, Klionsky Y, Rosen C, Bitcover R, Eventov-Friedman S, Katchman H, Cohen S, Tal O, Milstein O, Yagita H, Blazar BR, Reisner Y: Pig embryonic pancreatic tissue as a source for transplantation in diabetes: transient treatment with anti-LFA1, anti-CD48 and FTY720 enables long-term graft maintenance in mice with only mild ongoing immune suppression. Diabetes 2009, 58:1585-1594

29. Begum S, Chen W, Herold KC, Papaioannou VE: Remission of type 1 diabetes after anti-CD3 antibody treatment and transplantation of embryonic pancreatic precursors. Endocrinology 2009, 150:4512-4520

30. Korsgren O, Wallgran AC, Satake M, Karlsson-Parra A: Xenograft rejection of fetal porcine islet-like cell clusters in the rat: effect of active and passive immunization. Xenotransplantation 1999, 6:271-280

31. Soderlund J, Wennberg L, Castanos-Velez E, Biberfeld P, Zhu S, Tibell A, Groth CG, Korsgren O: Fetal porcine islet-like cell clusters transplanted to cynomolgous monkeys: an immunohistochemical study. Transplantation 1999, 67:784-791

32. Mandel TE: Fetal islet xenotransplantation in rodents and primates. J Mol Med 1999, 77:155-160

33. Dufrane D, Goebbels RM, Saliez A, Guiot Y, Gianello P: Six-month 
survival of microencapsulated pig islets and alginate biocompatibility in primates: proof of concept. Transplantation 2006, 81:1345-1353

34. Korsgren O: Acute cellular xenograft rejection. Xenotransplantation 1997, 4:11-19

35. Ryan EAQ, Lakey JRT, Rajotte RV, Korbutt GS, Kin T, Imes S, Rabinovich A, Elliott JF, Bigam D, Kneteman NM, Warnock GL, Larsen I, Shapiro AMJ: Clinical outcomes and insulin secretion after islet transplantation with the Edmonton protocol. Diabetes 2001, 50:710-719

36. Porta C, Rimoldi M, Raes G, Brys L, Ghezzi P, Di Liberto D, Dieli F,
Ghisletti S, Natoli G, De Baetselier P, Mantovani A, Sica A: Tolerance and M2 (alternative) macrophage polarization are related processes orchestrated by p50 nuclear factor $\kappa$ B. Proc Natl Acad Sci USA 2009, 106:14978-14983

37. Schroeder RA, Marroquin CE, Kuo PC: Tolerance and the "Holy Grail" of transplantation. J Surg Res, 2003, 111:109-119

38. Abraham EJ, Kodama S, Lin JC, Ubeda M, Raustman DL, Habener JF: Human pancreatic islet-derived progenitor cell engraftment in immunocompetent mice. Am J Pathol 2004, 164:817-830 